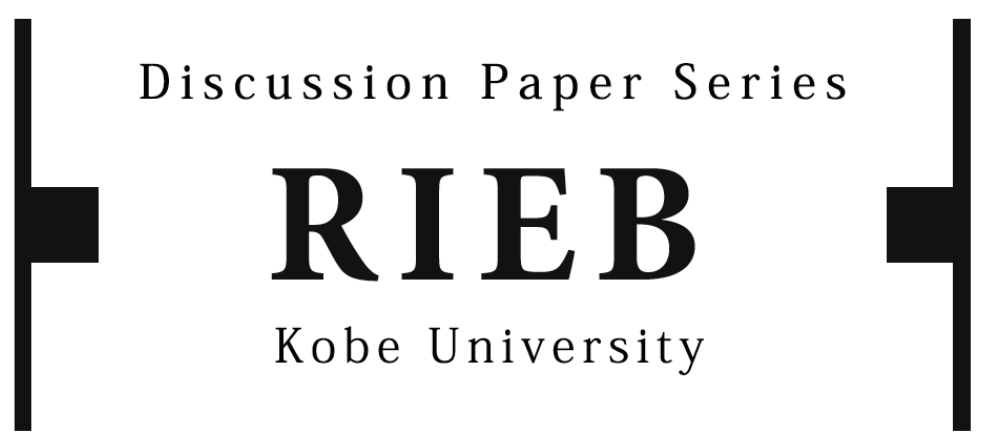

DP2020-17

Implementation in Iterative Elimination of Obviously Dominated Strategies: An Experiment on King Solomon's Dilemma*

\author{
Makoto HAGIWARA \\ Fumihiro YONEKURA
}

April 20, 2020

* The Discussion Papers are a series of research papers in their draft form, circulated to encourage discussion and comment. Citation and use of such a paper should take account of its provisional character. In some cases, a written consent of the author may be required.

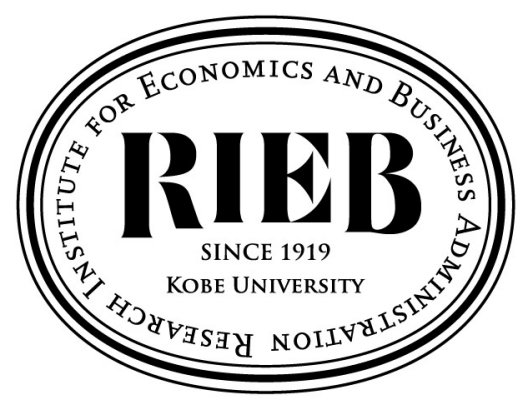

Research Institute for Economics and Business Administration

Kobe University

2-1 Rokkodai, Nada, Kobe 657-8501 JAPAN 


\title{
Implementation in Iterative Elimination of Obviously Dominated Strategies: An Experiment on King Solomon's Dilemma*
}

\author{
Makoto Hagiwara ${ }^{\dagger} \quad$ Fumihiro Yonekura ${ }^{\ddagger}$
}

April 20, 2020

\begin{abstract}
"King Solomon's Dilemma" is based on a biblical story and this can be considered as an allocation problem for an indivisible object among two players. A social planner wants to assign the object without payment to the player whose valuation is the highest. We say that such an allocation is "first-best." We experimentally compare the relative performance of the mechanism of Mihara (Japanese Economic Review, 63(3), 420 - 429, 2012) and a mechanism which we modify Mihara's mechanism. We find that a modified Mihara's mechanism relatively works better than Mihara's mechanism from the following five view points: (1) the proportion of the first-best allocations; (2) the proportion of the right-player allocations; (3) resource inefficiency and wrong-player inefficiency; (4) net mean efficiency; and (5) players' behavior.
\end{abstract}

JEL Classification: C92; D44; D78

Keywords: Implementation in iterative elimination of obviously dominated strategies; King Solomon's Dilemma; Mihara's mechanism; Ascending clock auctions; Laboratory experiment

*The authors are grateful to Emiko Fukuda, Ryo Kawasaki, Akiyoshi Shioura, Hirofumi Yamamura, and Takehiko Yamato for their helpful comments. Hagiwara was partially supported by JSPS KAKENHI Grant Number JP17J01520.

${ }^{\dagger}$ Corresponding author: Faculty of Economics, Osaka University of Economics, 2-2-8, Osumi, Higashiyodogawa-ku, Osaka, 533-8533, Japan; E-mail: m.hagiwara@osaka-ue.ac.jp

$\ddagger$ Department of Industrial Engineering and Economics, School of Engineering, Tokyo Institute of Technology, 2-12-1 Ookayama, Meguro-ku, Tokyo, 152-8552, Japan; E-mail: fumi314@yahoo.ne.jp 


\section{Introduction}

"King Solomon's Dilemma" is based on the biblical story in which King Solomon must give a child to one of two women who claim to be the true mother. The King and both women know that only one of the two women is the true mother. The King does not know which the right mother is. On the other hand, both women know who the true mother is, but the false mother does not want to disclose her true identity. The King wants to give the child to the true mother without payment. This problem is considered as an allocation problem for an identical and indivisible object among two players, and there are several examples of which this model is a realistic description (see Glazer and Ma, 1989; Footnote 1). ${ }^{1}$ A social planner wants to assign the good without payment to the player whose valuation is the highest. We say that such an allocation is "first-best."

Since the problem involves a transfer of money, an auction itself does not solve it (see Glazer and Ma, 1989; Moore, 1992; Perry and Reny, 1999). Several mechanisms have been proposed in order to solve the allocation problem.

Glazer and Ma (1989) and Moore (1992) assume that each player knows the other player's valuation. Under this information setting, they construct multi-stage mechanisms that implement the first-best allocation in subgame perfect equilibrium. Although those mechanisms consist of more than two stages, they have an appealing feature that only one player moves at each stage.

On the other hand, the four studies of Perry and Reny (1999), Olszewski (2003), Qin and Yang (2009), and Mihara (2012) assume that each player does not know the other player's valuation but knows her own valuation and whether she is the top valuation player. Perry and Reny (1999) and Olszewski (2003) construct mechanisms that implement the first-best allocation in iterative elimination of weakly dominated strategies. Qin and Yang (2009) design a mechanism that implement the first-best allocation in one round of elimination of weakly dominated strategies, followed by at most four rounds of iterative elimination of strictly dominated strategies. In contrast to these mechanism, Mihara (2012) provides a mechanism whose advantage is that only the top valuation player chooses to participate in the auction, which rules out the need to hold the auction. Mihara's mechanism consists of the following two stages: At the first stage, each player chooses whether she participates in the second-price auction or not: At the second stage, if both players choose to participate in the auction, all players pay a fee and partici-

\footnotetext{
${ }^{1}$ King Solomon's Dilemma is generalized by Qin and Yang (2009) as the allocation problem for $k$ identical and indivisible objects among at least two players. Mihara (2012) also investigates this generalized problem.
} 
pate the second-price auction. Mihara (2012) assumes that the players and the planner know that there is a gap between the two players' valuations which is greater than $M$. Mihara's mechanism implements the first-best allocation in one round of elimination of weakly dominated strategies, followed by two rounds of iterative elimination of strictly dominated strategies.

For the result of Mihara (2012), an important feature of the second-price auction is "strategy-proofness." This axiom states that in the direct revelation mechanism, truthtelling is a weakly dominant strategy for each player. Even though choosing when to quit in an ascending clock auction is the same as choosing a bid in the second-price auction, ascending clock auctions are simpler for real people to understand than the second-price auction. As evidence from a laboratory experiment, subjects are substantially more likely to play the dominant strategy under an ascending clock auction than under the second-price auction (Kagel, Harstad, and Levin, 1987). Inspired by this observation, Li (2017) models what it means by "obvious dominance." This definition distinguishes ascending clock auctions and the second-price auction. Ascending clock auctions are obviously strategy-proof. On the other hand, the second-price auction is strategy-proof, but not obviously strategy-proof. Therefore, in iterative elimination of obviously dominated strategies, Mihara's mechanism does not implement the first-best allocation.

We modify Mihara's mechanism as changing the second-price auction into an ascending clock auction. This modified mechanism implements the first-best allocation in iterative elimination of obviously dominated strategies (Proposition 2). This result is shown, together with Table 1 of Mihara (2012), by the reason that in an ascending clock auction, for each player, quitting when the price is her own valuation is the unique obviously dominant strategy.

A bunch of experimental studies suggest that players perform only a few number of steps of iterative elimination of dominated strategies (Nagel, 1995; Sefton and Yavas, 1996; Costa-Gomes, Crawford, and Broseta, 2001; Katok, Sefton, and Yavas, 2002; Rapoport and Amaldoss, 2000; Kneeland, 2015). While there is evidence that it is hard to apply the notion of implementation in iterative elimination of dominated strategies to real people, the result of Masuda, Okano, and Saijo (2014) suggests that the notion of implementation in iterative elimination of obviously dominated strategies is not the case. $^{2}$ They report that in the public good provision, the mechanism which implements the Pareto-efficient allocation in iterative elimination of obviously dominated strategies

\footnotetext{
${ }^{2}$ Note that Masuda, Okano, and Saijo (2014) do not state the feature of implementation of iterative elimination of obviously dominated strategies in their mechanism.
} 
works well. In their laboratory experiment, players simultaneously and independently choose their contributions to the public good from 0 to 24 as integers. Moreover, in each mechanism designed by them, players simultaneously and independently decide whether to approve the other's choice or not. Then, the number of strategies in their experiment for each player is $25 \times 2=50$. On the other hand, as in Section 3, the number of strategies in our experiment for each player is $2 \times 271=542$. Even though the number of strategies for each player is greater, our mechanism works well.

We experimentally compare the relative performance of Mihara's mechanism and our mechanism. Our experiment find the following five observations: (1) Our mechanism attains the first-best allocation more frequently than Mihara's mechanism (Result 1); (2) Our mechanism allocates the object to the right player more frequently than Mihara's mechanism (Result 2) ${ }^{3}$; (3) Mihara's mechanism is more inefficient than our mechanism from the view point of resource inefficiency and right-player inefficiency (Results 3 and 4); (4) Net mean efficiency under our mechanism was $91.3 \%$, while net mean efficiency under Mihara's mechanism was $71.8 \%$ (see Table 2); and (5) The equilibrium strategies are frequently taken under our mechanism than under Mihara's mechanism (see Subsection 4.3). In addition, we compare the results of Elbittar and Di Giannatale (2017) with our results under the same setting as ours. ${ }^{4}$ Our mechanism works better than any mechanisms investigated by Elbittar and Di Giannatale (2017).

The rest of the paper is organized as follows. Section 2 provides the model and two mechanisms. Section 3 describes our experimental design. Section 4 reports our results and provides discussion. Section 5 summarizes our results and further researches as a conclusion.

\section{The Model and Two Mechanisms}

Suppose that there are only two players, 1 and 2. A single, identical, and indivisible object is to be allocated to the player whose valuation is the highest. It is common knowledge that one player's valuation exceeds the other's. Each player knows her own valuation, and whose valuation is higher. A social planner knows neither players' valuations nor

\footnotetext{
${ }^{3}$ For both mechanisms, the first-best allocations and the right-player allocations are more frequently achieved in the latter half than in the first half.

${ }^{4}$ Ponti, Gantner, López-Pintado, and Montgomery (2003) also conducted an experiment for King Solomon's Dilemma. While they considered the only five cases of payoff vectors that the two players have, both this paper and Elbittar and Di Giannatale (2017) investigate much more cases of payoff vectors and each case is randomly chosen by computer. Then, it is hard to compare directly the results of Ponti, Gantner, López-Pintado, and Montgomery (2003) with ours nor the results of Elbittar and Di Giannatale (2017).
} 
whose valuation is higher. Each player's payoff of obtaining the object and payment $p$ is $v+p$, where $v$ is the player's valuation of the object. Each player's payoff of obtaining no object and payment $p$ is $p$. We assume that the two players and the planner know that there is a gap between the highest and the second highest valuations which is greater than a positive real number $M>0$. The planner wants to allocate the object without payment to the player whose valuation is the highest. Hereafter, we say that such an allocation is first-best.

For the allocation problem, the following mechanism is designed by Mihara (2012):

The Second-Price Auction with the Participation Stage (hereafter, SPAPS)

The participation stage: Both two players simultaneously saying either "auction" or "no auction."

If only one player says "auction," then this player is eligible to acquire the object without payment and the other player gets and pays nothing, and the game ends. If no players say "auction," then the object is assigned randomly, and the game ends. If both two players say "auction," then we move to the second-price auction after each player pays the participation fee, $M$.

The auction stage: Both two players simultaneously and independently offer bids for the object. The player whose bid is the highest is eligible to acquire the object at the price that the other player offers a bid and the other player gets and pays nothing except for the participation fee, and the game ends.

Regarding SPAPS, Mihara (2012) provides the following result:

Proposition 1. (Mihara, 2012) SPAPS implements the first-best allocation in one round of elimination of weakly dominated strategies, followed by two rounds of iterative elimination of strictly dominated strategies

Strategy-proofness requires that in the direct revelation mechanism, truth-telling is a weakly dominant strategy for each agent. ${ }^{5}$ While the second-price auction is strategyproof, it is not obviously strategy-proof. ${ }^{6}$ Hence, SPAPS does not implement the first-best allocation in iterative elimination of obviously dominated strategies. Then, we modify SPAPS as follows:

\footnotetext{
${ }^{5}$ For the definition of strategy-proofness, see for example Barberà (2011).

${ }^{6} \mathrm{~A}$ strategy is obviously dominant if, for any deviation, at any information set where both strategies first diverge, the best outcome under the deviation is no better than the worst outcome under the dominant strategy. A mechanism is obviously strategy-proof if it has an equilibrium in obviously dominant strategies. For the formal definitions of obviously dominant strategies and obvious strategy-proofness, see Li (2017).
} 
An Ascending Clock Auction with the Participation Stage (hereafter, ACAPS) The participation stage: This stage is the same as Mihara's mechanism.

The auction stage: The price of the object begins at 0 and increases the price for the object. Each participant will be regarded as an active bidder for the object until the player cease to bid on the object. Each player can drop out of the auction. Exit from the auction is not reversible i.e., no player can re-enter once she is out. The only one player who is still an active bidder, i.e. did not drop out, is eligible to acquire the object at the price at which the other bidder exited.

Since an ascending clock auction is obviously strategy-proof, together with Table 1 of Mihara (2012), we have the following result:

Proposition 2. ACAPS implements the first-best allocation in iterative elimination of obviously dominated strategies.

The idea to have Proposition 2 is not difficult. Suppose that at the participation stage, each player says "auction." In the auction stage, for each player, quitting when the price is her own valuation is the unique obviously dominant strategy. Given this remaining strategy profile, saying "auction" is the unique obviously dominant strategy for the player whose valuation is the highest. Given this remaining strategy profile in the auction stage and this remaining strategy of the player whose valuation is the highest, saying "no auction" is the unique obviously dominant strategy for the player whose valuation is the lowest. Therefore, the unique remaining strategy profile from iterative elimination of obviously dominated strategies is that the player whose valuation is the highest says "auction" and the player whose valuation is the lowest says "no auction." Therefore, by these three steps, we have the statement of Proposition 2.

\section{Experimental Design}

Our experiment compares the relative performance of SPAPS and ACAPS. All settings regarding our experimental design are the same as those of Elbittar and Di Giannatale (2017) except the amount of the entry payment for our experiment and the number of the practice and real rounds. To compare our results with theirs, we use the almost same settings and the reasons for three different settings are discussed in Footnotes 8 and 10.

The details in the experimental design are as follow.

Subjects. For each session, the subjects were drawn from a wide of undergraduate 
and graduate students at Tokyo Institute of Technology. The subjects were told that there would be an opportunity to earn money in our experiment. None of them had prior experience in a second-price-auction experiment nor an ascending-clock-auction experiment nor a King-Solomon-Dilemma experiment.

Two sessions were conducted under each of SPAPS and ACAPS, and 20 subjects participated in each session (80 separate subjects in total). No subject attended more than one session. Our experiment was conducted at Tokyo Institute of Technology during December of 2019 using computers with the experimental software, z-Tree (Fischbacher, 2007). In each session, 20 subjects were seated at computer stations that were separated with visual partitions in the Experimental Economics Laboratory at Tokyo Institute of Technology.

Flow of the Experiment. Upon subjects' arrival, they were randomly assigned to a computer terminal. Each subject received an instruction, a post-experimental questionnaire, and a record sheet which all are in Japanese, together with writing materials such as two pencils. ${ }^{7}$ Each instruction explains the summary of our experiment, the details of our experiment, the procedures of our experiment, and how to decide points and payments. After all subjects were seated and the experimenter confirmed that subjects received all experimental materials, the instruction was read aloud by the experimenter for subjects to understand the instruction equally. Subjects were allowed to ask any question they had during the whole process. After reading the instruction, our experiment was initiated by computer. At the end of our experiment, each participant answered a questionnaire and then the payments obtained by subjects were made by cash.

Practice and Real Periods. In order to familiarize the subjects with the procedures, 3 practice periods and 20 real periods. ${ }^{8}$

Matching Procedure. After the three practice periods, each subject was designated either as a High Valuation Player (hereafter, HVP) or a Low Valuation Player (hereafter, LVP). These roles remained fixed for the whole session, so that each type of a player identifies herself with her role. In each period, an HVP was paired with an LVP, and each pairing was randomized. They were never paired with the same subject more than twice,

\footnotetext{
${ }^{7}$ In the following website, see the instructions, the post-experimental questionnaire, and the record sheets in English that are translated from Japanese and in Japanese that are used in our experiment: https://sites.google.com/view/makotohagiwara-economics/research

${ }^{8}$ In contrast to ours, Elbittar and Di Giannatale (2017) conducted the 2 practice periods and the 10 real periods. We changed the settings because we would like for subjects to understand all cases of SPAPS or ACAPS and also find learning effect.
} 
and were never paired with the same subject in two consecutive periods. Furthermore, they did not know who they were paired with in any given period.

Valuations. Let $\theta_{H V P}$ be the valuation of an HVP and $\theta_{L V P}$ be the valuation of an LVP. Players' valuations for each period were drawn randomly from the interval $[0,200]$ as integers, with the restriction that the difference between $\theta_{H V P}$ and $\theta_{L V P}$ would be more than $M \equiv 50$ :

$$
\theta_{H V P}-\theta_{L V P}>M \equiv 50 .
$$

Information Setting. We consider the following information setting: both players were informed of the ranking of their valuations i.e., whether they had the higher or lower valuation, but they were not informed of the exact amount of the other player's valuation. Under this information setting, each player's screen showed her own valuation, but not the valuation of the player with whom was paired. However, the players knew that the difference between the players' valuations was more than 50 francs.

Initial Capital. All players were endowed with an initial capital balance in according to the role in the conducted period. The initial capital balances were 30 francs for the HVPs and 70 francs for the LVPs. ${ }^{9}$ The reason for the difference between the initial capital of the LVPs and that of the HVPs was to compensate for the asymmetry in their valuations and deter envy-driven actions, as in Elbittar and Di Giannatale (2017).

Information Feedback. During the decision process, some of the calculations about possible payoffs were given privately by the computer to each subject at different decision nodes. After participating an auction and the bids were submitted for the second-price auction, or the first drop-out occurred for an ascending clock auction, the payoffs conditional on keeping or giving up the object were calculated for the winner. Finally, at the end of each round, each subject received complete feedback about her own payoff.

Payoffs and Final Payments. The final payoff was determined selecting one round randomly out of the 20 real periods played for money. Subjects were also informed that any winnings would be added to the initial capital balance, and any losses would be subtracted from it. The initial capital balance, plus gains or minus losses, was considered their possible payoff for each period. The equilibrium expected payoffs were 193 francs for the HVPs and 70 francs for the LVPs, as in Elbittar and Di Giannatale (2017).

Under SPAPS, the final payment on average was 3054 Japanese yen, and the experimental time was about 2 hours and a half. Under ACAPS, the final payment on average

\footnotetext{
${ }^{9}$ Regarding the initial capital balances, we will discuss those in Section 5.
} 
was 3719 Japanese yen, and the experimental time was about 3 hours. ${ }^{10}$ Note that the experimental time was different between SPAPS and ACAPS due to the waiting time in an ascending-clock auction.

Bidding Restrictions. For the ascending clock auction, subjects were informed that the clock price had reached the maximum level of 270 without a subject dropping out, the sale price for the item would be 270 and the item would be sold to one of the claimants (chosen randomly by computer) at this price, without the option to withdraw, and the other bidder would pay nothing. In order to compare an ascending-clock auction with the second-price auction, subjects were not allowed to bid above 270. This restriction was imposed to avoid allowing the subjects to incur losses.

\section{Results and Discussion}

\subsection{Efficiency}

To compare the results of our experiment with the results of Elbittar and Di Giannatale (2017), we consider the two different measures of "efficiency:" (1) the proportion of first-best allocations, and (2) the proportion of right-player allocations. The first is obtained by dividing the number of objects given to HVPs without payment by the total number of allocations. The second is obtained by dividing the number of objects given to HVPs with or without payment by the total number of allocations.

Figure 1 (resp. Figure 2) reports the transition of the proportions of each of the firstbest allocations and the right-player allocations under SPAPS (resp. ACAPS). Figure 3 reports the aggregate proportions of the first-best allocations and right-player allocations under SPAPS and ACAPS. From these figures, the performance regarding each of the first-best allocations and the right-player allocations under ACAPS is better than under SPAPS.

To investigate these observation statistically, we conduct pairwise z-tests between SPAPS and ACAPS (see Table 1). ${ }^{11}$

The following are our two results:

Result 1. (First-best allocations) The ratio of the first-best allocations under ACAPS

\footnotetext{
${ }^{10}$ While Elbittar and Di Giannatale (2017) used the payment of 50 francs for each subject as an entry payment in their experiment, we payed 1013 Japanese yen (or 506.5 francs) hourly. The amount of 1013 Japanese yen is decided for the compensation of the minimum wage in Tokyo, Japan in 2019 which is irrelevant to the performances of subjects. See the following URL:

https://pc.saiteichingin.info/kouho/pdf/leaflet/english/13_mw2019_A4_english_toukyou.pdf

${ }^{11}$ For pairwise z-tests, we essentially follow Ponti, Gantner, López-Pintado, and Montgomery (2003) (see Footnote 19 on page 225 of their paper).
} 


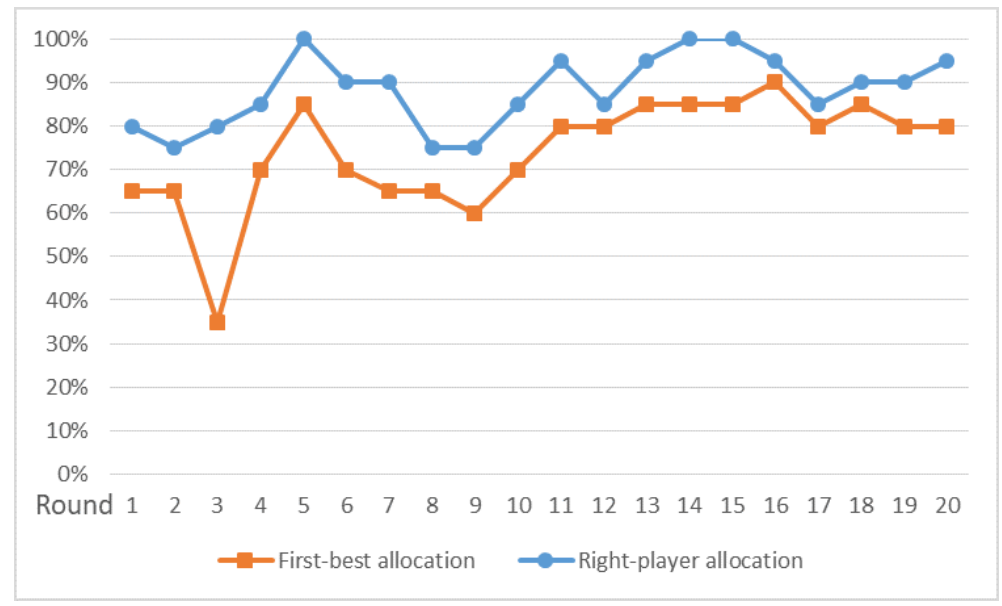

Figure 1: Transition of the proportion of each of the first-best and the right-player allocations under SPAPS.

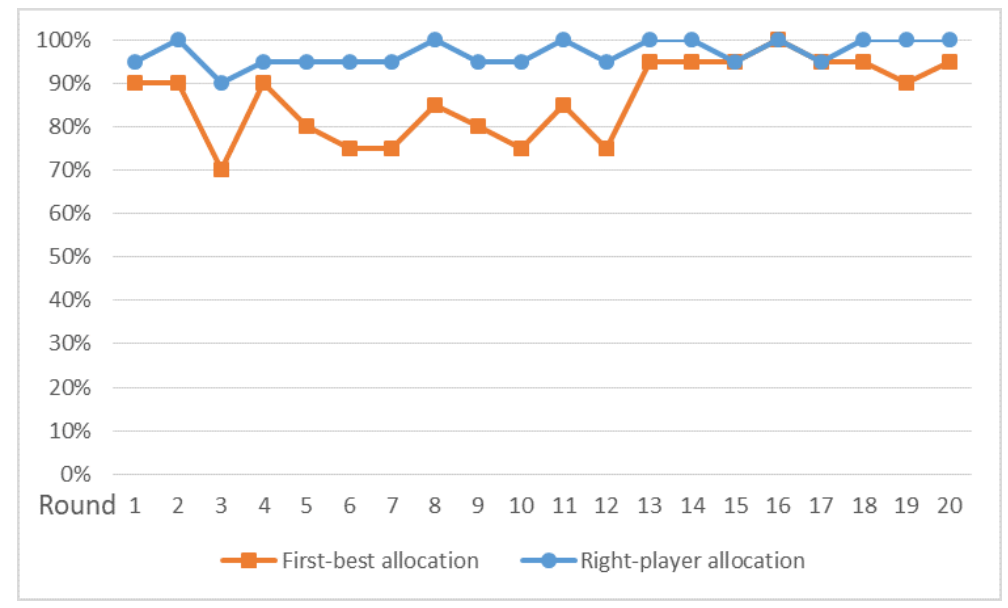

Figure 2: Transition of the proportion of each of the first-best and the right-player allocations under ACAPS.

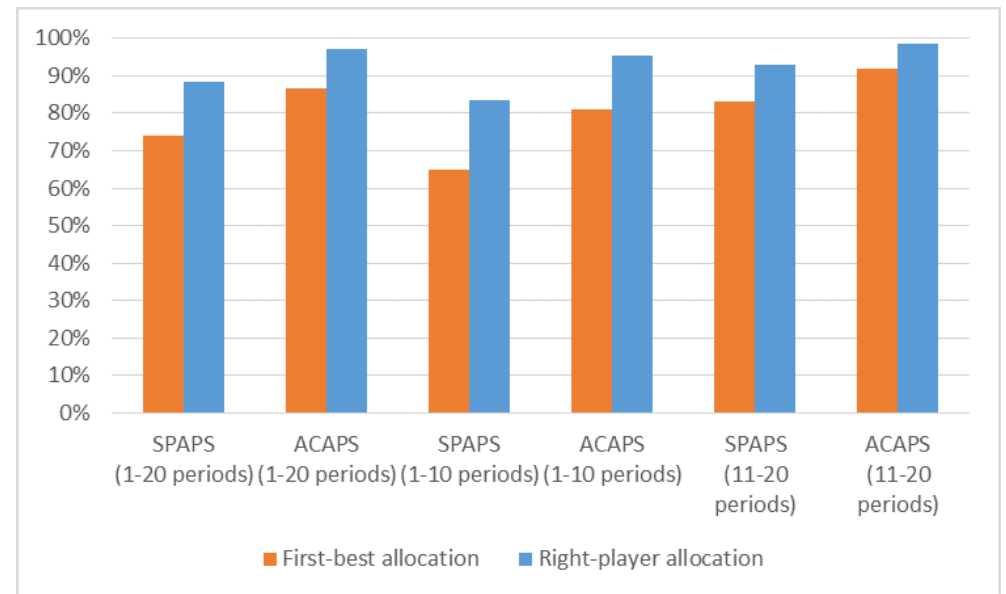

Figure 3: Aggregate proportions of each of the first-best allocations and right-player allocations under SPAPS and ACAPS. 
Table 1: Aggregate proportions of allocations for SPAPS and ACAPS.

\begin{tabular}{|c|c|c|c|c|c|c|}
\hline & \multicolumn{2}{|c|}{ 1-20 periods } & \multicolumn{2}{|c|}{$1-10$ periods } & \multicolumn{2}{|c|}{$11-20$ periods } \\
\hline & SPAPS & ACAPS & SPAPS & ACAPS & SPAPS & ACAPS \\
\hline (1) HVPs get the object & 0.88 & $0.97^{* * *}$ & 0.84 & $0.96^{* * *}$ & 0.93 & $0.99^{* *}$ \\
\hline (1a) without payment & 0.74 & $0.87^{* * *}$ & 0.65 & $0.81^{* * *}$ & 0.83 & $0.92^{* *}$ \\
\hline (1b) with payment & 0.14 & 0.11 & 0.19 & 0.15 & 0.10 & 0.07 \\
\hline (2) LVPs get the object & 0.10 & $0.02^{* * *}$ & 0.14 & $0.04^{* * *}$ & 0.07 & $0.01^{* *}$ \\
\hline (2a) without payment & 0.02 & $0.00^{* *}$ & 0.03 & $0.00^{*}$ & 0.01 & 0.00 \\
\hline (2b) with payment & 0.08 & $0.02^{* * *}$ & 0.11 & $0.04^{* *}$ & 0.06 & $0.01^{* *}$ \\
\hline (3) No one gets the object & 0.02 & 0.01 & 0.03 & 0.01 & 0.01 & 0.01 \\
\hline Observations & 400 & 400 & 200 & 200 & 200 & 200 \\
\hline
\end{tabular}

Note: ${ }^{*} p<0.05 ;{ }^{* *} p<0.01 ;{ }^{* * *} p<0.001$ 
is significantly larger than under SPAPS for total 20 periods, $1-10$ periods, and $11-20$ periods, respectively.

Support. From (1a) in Table. 1, the aggregate proportion of allocations for total 20 periods (resp. $1-10$ periods, $11-20$ periods) when HVPs get the object under SPAPS without payment is $0.74=296 / 400$ (resp. $0.65=130 / 200,0.83=166 / 200$ ), and that for ACAPS is $0.87=346 / 400$ (resp. $0.81=162 / 200,0.92=184 / 200$ ). For each of total 20 periods, $1-10$ periods, and $11-20$ periods, since the latter proportion is greater than the former proportion, ACAPS performs better than SPAPS. In addition, the difference is statistically significant at the $0.1 \%$-level (resp. $0.1 \%, 1 \%$ ).

Result 2. (Right-player allocations) The ratio of the right-player allocations under $A C A P S$ is significantly larger than under SPAPS for total 20 periods, $1-10$ periods, and $11-20$ periods, respectively.

Support. From (1) in Table. 1, the aggregate proportion of allocations for total 20 periods (resp. $1-10$ periods, $11-20$ periods) when HVPs get the object under SPAPS with or without payment is $0.88=353 / 400$ (resp. $0.84=167 / 200,0.93=186 / 200$ ), and that for ACAPS is $0.97=388 / 400$ (resp. $0.96=191 / 200,0.99=197 / 200$ ). For each of total 20 periods, $1-10$ periods, and $11-20$ periods, since the latter proportion is greater than the former proportion, ACAPS performs better than SPAPS. In addition, the difference is statistically significant at the $0.1 \%$-level (resp. $0.1 \%, 1 \%$ ).

From these results, we observed that ACAPS achieved both the first-best allocations and the right-player allocations more frequently than SPAPS for total 20 periods, $1-10$ periods, and 11-20 periods, respectively. In addition, for both mechanisms, the first-best allocations and the right-player allocations were more frequently achieved in the latter half than in the first half.

We compare our experimental results under SPAPS and ACAPS and those of Elbittar and Di Giannatale (2017) regarding the following three mechanism: Perry and Reny (1999) mechanism (hereafter, PRM), PRM with an ascending clock auction (hereafter, PRMACA), and PRM with a slow ascending clock (hereafter, PRMSACA). ${ }^{12}$

Figure 4 provides the comparison of the experimental results under these five mechanisms. Note that since Elbittar and Di Giannatale (2017) conducted their experiments

\footnotetext{
${ }^{12}$ Note that the experiment of Elbittar and Di Giannatale (2017) was conducted in Mexico City, while our experiment was run in Tokyo, Japan.
} 


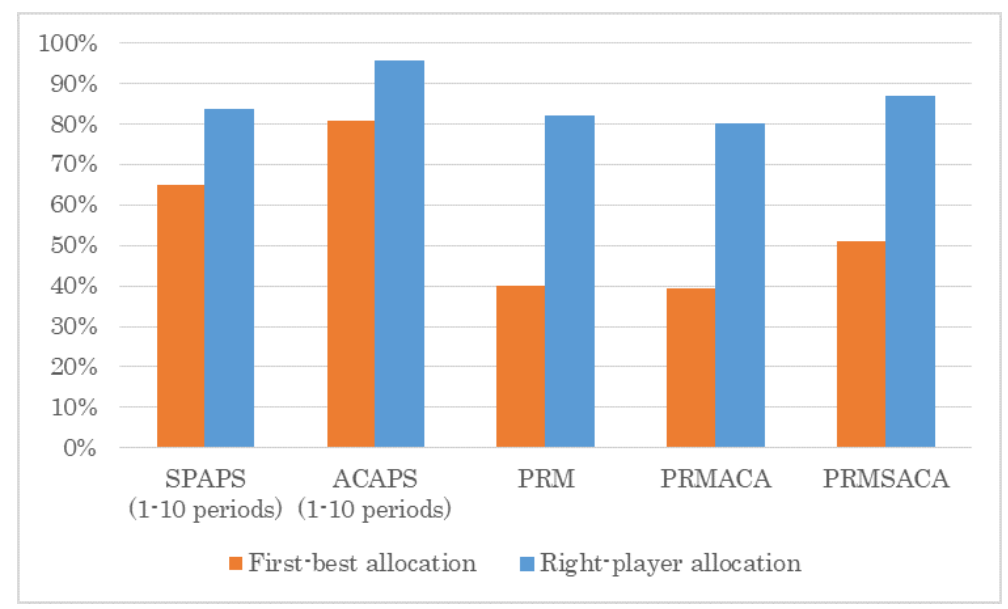

Figure 4: Comparison with SPAPS, ACAPS, PRM, PRMACA and PRMSACA.

for only $1-10$ periods, we also focus on $1-10$ periods. As in Figure 4, ACAPS attains both the first-best allocations and the right-player allocations more frequently than PRM, PRMACA and PRMSACA. The aggregate proportions of the first-best allocations under SPAPS, ACAPS, PRM, PRMACA and PRMSACA are 65\%, 81\%, 40\%, 39\% and $51 \%$, respectively. The aggregate proportions of the right-player allocations under SPAPS, ACAPS, PRM, PRMACA and PRMSACA are 84\%, 96\%, 82\%, $80 \%$ and 87\%, respectively.

\section{$4.2 \quad$ Sources of Inefficiency}

To compare our experimental results with those of Elbittar and Di Giannatale (2017), we consider two possible sources of inefficiency: (1) wrong-player inefficiency, and (2) resource inefficiency. The first is obtained by dividing the high valuation minus winner's valuation by the summation of the high valuation and the initial capitals of an HVP and an LVP. The second is obtained by dividing the summation of the two players' payments by the summation of the high valuation and the two players' initial capitals. In other words, the proportion of wrong-player inefficiency tells us the inefficiency from allocating the object to an LVP, and the proportion of resource inefficiency tells us the inefficiency of monetary resources from paying the participation fee and the winning bid.

Before analyzing these two sources of inefficiency, let us introduce the notion of net mean efficiency as the total amount of players' net gains as a proportion of the total surplus. At equilibrium, the net mean efficiency should be a hundred percent of the total surplus, otherwise there would be two types of inefficiency: wrong-player inefficiency and resource inefficiency. Table 2 reports the aggregate proportions of net mean effi- 
Table 2: Aggregate proportions of Net Mean Efficiency (NME), Resource Inefficiency (RI), and Wrong-Player Inefficiency (WPI).

\begin{tabular}{lccc}
\hline & Net Mean Efficiency $^{a}$ & Resource Inefficiency $^{b}$ & Wrong-Player Inefficiency $^{c}$ \\
\hline SPAPS $^{d}$ & $71.8 \%$ & $23.0 \%$ & $5.2 \%$ \\
ACAPS $^{d}$ & $91.3 \%$ & $7.2 \%$ & $1.5 \%$ \\
\hline PRM & $57.4 \%$ & $35.9 \%$ & $6.7 \%$ \\
PRMACA & $70.1 \%$ & $23.4 \%$ & $6.5 \%$ \\
PRMSACA & $75.0 \%$ & $21.2 \%$ & $3.8 \%$ \\
\hline
\end{tabular}

${ }^{a} \mathrm{NME}=($ Winner's valuation-Players' payments+Players' initial capital $)$ /(Highest valuation+Players' initial capital)

${ }^{b} \mathrm{RI}=$ Players' payments/(Highest valuation+Players' initial capital)

${ }^{c}$ WPI $=($ Highest valuation - Winner's valuation $) /($ Highest valuation+Players' initial capital)

${ }^{d}$ In order to compare with Elbittar and Di Giannatale (2017), the aggregate proportion is for $1-10$ periods.

ciency, wrong-player inefficiency and resource inefficiency under SPAPS, ACAPS, PRM, PRMACA, and PRMSACA. ${ }^{13}$ From Table 2, resource inefficiency is the main source of inefficiency under each of all five mechanisms, and ACAPS achieves higher net mean efficiency than the other four mechanisms.

We measure how players' valuations and a different mechanism affect our sources of inefficiency. In the following Ordinary Least Squares (OLS) regression regarding each of wrong-player inefficiency and resource inefficiency, SPAPS is the baseline model and is compared to ACAPS. The specifications can be formulated, in general, as follows:

$$
y_{i t}=\text { intercept }+\beta_{1} \theta_{H V P, i t}+\beta_{2} \theta_{L V P, i t}+\beta_{3} p e r+\gamma d_{A C A P S}+v_{i}+\epsilon_{i t},
$$

where $y_{i t}$ represents the proportion of either wrong-player inefficiency or resource inefficiency for player $i$ at period $t ; \theta_{H V P, i t}$ represents the valuation of the HVP for player $i$ at period $t ; \theta_{L V P, i t}$ represents the valuation of the LVP for player $i$ at period $t$; per represents the period treating time as a continuous variable; $d_{A C A P S}$ represents a dummy variable that takes the value of one in the case of ACAPS; $v_{i}$ is the individual-specific error term and $\epsilon_{i t}$ is the usual error term assumed to be i.i.d. for player $i$ at period $t$. Table 3 provides the regression results for sources of inefficiency.

\footnotetext{
${ }^{13}$ The aggregate proportions of net mean efficiency, wrong-player inefficiency and resource inefficiency
} 
Table 3: Regression results for sources of inefficiency.

\begin{tabular}{lcc}
\hline \hline & Resource Inefficiency & Wrong-Player Inefficiency \\
\hline$\theta_{H V P}$ & -0.0001 & $-0.0004^{* * *}$ \\
& $(0.0002)$ & $(0.0001)$ \\
$\theta_{L V P}$ & $0.003^{* * *}$ & $0.0002^{* * *}$ \\
& $(0.0002)$ & $(0.0001)$ \\
& $-0.007^{* * *}$ & $-0.002^{* * *}$ \\
per & $(0.001)$ & $(0.0004)$ \\
& $-0.082^{* * *}$ & $-0.033^{* * *}$ \\
$d_{A C A P S}$ & $(0.026)$ & $(0.007)$ \\
& $0.136^{* * *}$ & $0.111^{* * *}$ \\
Intercept & $(0.037)$ & $(0.012)$ \\
& & 1,600 \\
\hline Observations & 1,600 & \\
\hline \hline
\end{tabular}

Note: Numbers in parentheses below each coefficient represent the coefficient's standard error.

${ }^{*} p<0.05 ;{ }^{* *} p<0.01 ;{ }^{* * *} p<0.001$ 
The following are our two findings regarding sources of inefficiency:

\section{Result 3. (Resource inefficiency)}

1. The proportion of the inefficiency due to the use of monetary resources in the allocation process in ACAPS is lower than in SPAPS.

2. When LVPs' valuations increase, the proportion of the inefficiency of the monetary resources increases.

3. As the period progresses, the proportions of the inefficiency of the monetary resources under SPAPS and ACAPS decrease.

Support. 1. In Table 3, the coefficient for $d_{A C A P S}$ is negative $(-0.082)$ and the difference is statistically significant at $0.1 \%$-level, which indicates a reduction in the use of monetary resources under ACAPS.

2. The coefficient for $\theta_{L V P}$ is positive (0.003) and the difference is statistically significant at $0.1 \%-$ level.

3. The coefficient for per is negative $(-0.007)$ and the difference is statistically significant at $0.1 \%$-level.

\section{Result 4. (Wrong-player inefficiency)}

1. The proportion of the inefficiency for allocating the object to the wrong player under $A C A P S$ is lower than under SPAPS.

2. When HVPs' valuations decrease or LVPs'valuations increase, the proportions of the inefficiency for allocating the object to the wrong player decrease.

3. As the period progresses, the proportion of the inefficiency from allocating the object to the wrong player decreases.

Support. 1. In Table 3, the coefficient for $d_{A C A P S}$ is negative $(-0.033)$. In addition, the difference is statistically significant at $0.1 \%$-level, which indicates a reduction of cases that the object is allocated to the wrong player under ACAPS.

2. The coefficient for $\theta_{H V P}$ is negative $(-0.0004)$ and that for $\theta_{L V P}$ is positive (0.0002). For each of these coefficients, the difference is statistically significant at $0.1 \%$-level. 
Table 4: Players' behavior at the participation stage.

\begin{tabular}{lcc}
\hline & SPAPS & ACAPS \\
\hline (1) HVPs' decision & & \\
(1a) "auction" & $96.5 \%=772 / 800$ & $99.3 \%=794 / 800$ \\
(1b) "no auction" & $3.5 \%=28 / 800$ & $0.7 \%=6 / 800$ \\
(2) LVPs' decision & & \\
(2a) "auction" & $24.3 \%=194 / 800$ & $12.8 \%=102 / 800$ \\
(2b) "no auction" & $75.8 \%=606 / 800$ & $87.3 \%=698 / 800$ \\
\hline
\end{tabular}

3. The coefficient for per is negative $(-0.002)$, and the difference is statistically significant at $0.1 \%-$ level.

\subsection{Players' Behavior}

\subsubsection{The Participation Stage}

Table 4 describes the aggregate proportions of the players who say "auction" or "no auction" at the participation stage for total 20 periods under SPAPS and ACAPS. Under SPAPS, HVPs say "auction" at $96.5 \%$ and LVPs say "no auction" at $75.8 \%$. Under ACAPS, HVPs say "auction" at $99.3 \%$ and LVPs say "no auction" at $87.3 \%$. Therefore, the performance under ACAPS is better than under SPAPS at the participation stage. Figure 5 (resp. Figure 6) describes the transition regarding the aggregate proportion of the players who say "auction" at the participation stage for each period under SPAPS (resp. ACAPS). These figures seem to represent the tendency that as the period progresses, the equilibrium action at the participation stage for HVPs and LVPs under each mechanism is more frequently taken.

Regarding the above observations, we statistically measure how players' valuations and a different mechanism affect players' behavior regarding whether they participate in the auction or not. In the following logit regression regarding each of saying "auction" and saying "no auction," SPAPS is the baseline model and is compared to ACAPS. The specifications can be formulated, in general, as follows: For HVPs,

$$
y_{i t}^{\prime}=i n t e r c e p t+\beta_{1}^{\prime} \theta_{H V P, i t}+\beta_{2}^{\prime} p e r+\gamma^{\prime} d_{A C A P S}+v_{i}^{\prime}+\epsilon_{i t}^{\prime},
$$




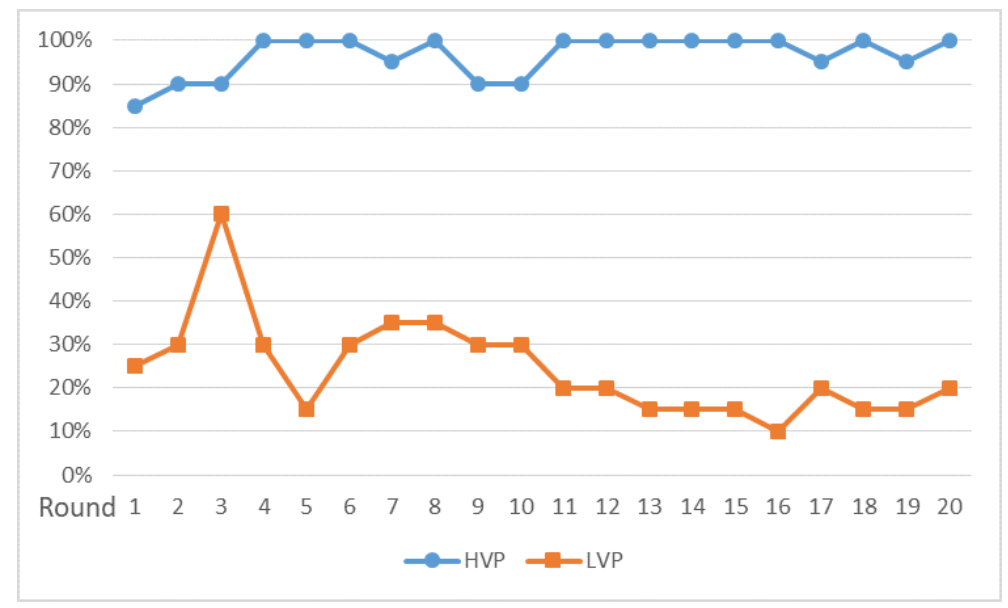

Figure 5: Participation rate for each period at the participation stage under SPAPS

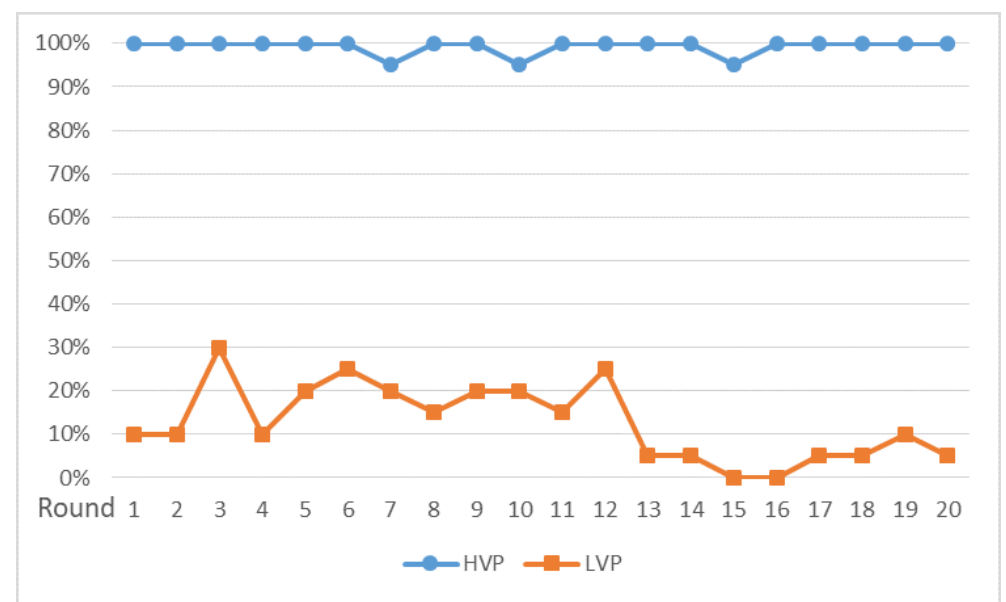

Figure 6: Participation rate for each period at the participation stage under ACAPS 
Table 5: Regression results for HVPs' behavior and LVPs' behavior at the participation stage.

\begin{tabular}{lcc}
\hline \hline & HVPs & LVPs \\
\hline$\theta_{H V P}$ & $\begin{array}{c}0.033^{* * *} \\
(0.005)\end{array}$ & - \\
& - & $0.024^{* * *}$ \\
$\theta_{L V P}$ & & $(0.002)$ \\
& & $-0.098^{* * *}$ \\
per & $0.095^{* *}$ & $(0.014)$ \\
& $(0.034)$ & $-1.098^{* * *}$ \\
$d_{A C A P S}$ & $1.724^{* * *}$ & $(0.292)$ \\
& $(0.464)$ & $-1.677^{* * *}$ \\
Intercept & $-1.896^{* *}$ & $(0.257)$ \\
& $(0.689)$ & 800 \\
\hline Observations & 800 & \\
\hline \hline
\end{tabular}

Note: Numbers in parentheses below each coefficient represent the coefficient's standard error.

${ }^{*} p<0.05 ;{ }^{* *} p<0.01 ;{ }^{* * *} p<0.001$

and for LVPs,

$$
y_{i t}^{\prime \prime}=\text { intercept }+\beta_{1}^{\prime \prime} \theta_{L V P, i t}+\beta_{2}^{\prime \prime} p e r+\gamma^{\prime \prime} d_{A C A P S}+v_{i}^{\prime \prime}+\epsilon_{i t}^{\prime \prime},
$$

where $y_{i t}^{\prime}$ (resp. $\left.y_{i t}^{\prime \prime}\right)$ represents either "auction" or "no auction" chosen by HPV (resp. LVP) $i$ at period $t$ and both $y_{i t}^{\prime}=1$ and $y_{i t}^{\prime \prime}=1$ (resp. $y_{i t}^{\prime}=0$ and $\left.y_{i t}^{\prime \prime}=0\right)$ mean that at period $t$, player $i$ says "auction" (resp. "no auction"); $v_{i}^{\prime}$ and $v_{i}^{\prime \prime}$ are the individual-specific error terms and $\epsilon_{i t}^{\prime}$ and $\epsilon_{i t}^{\prime \prime}$ are the usual error terms assumed to be i.i.d. for player $i$ at period $t$. Table 5 provides the regression results for HVPs' behavior and LVPs' behavior at the participation stage.

The following are our two findings regarding players' behavior at the participation stage:

\section{Result 5. (HVPs' behavior at the participation stage)}

1. Saying "auction" at the participation stage for HVPs under ACAPS is more frequently taken than under SPAPS. 
2. When HVPs' valuations increase, saying "auction" at the participation stage is more frequently taken.

3. As the period progresses, saying "auction" at the participation stage for HVPs is more frequently taken.

Support. $\quad$ 1. In Table 5, the coefficient for $d_{A C A P S}$ is positive (1.724) and the difference is statistically significant at $0.1 \%$-level, which indicates the increase that HVPs say "auction" under ACAPS.

2. The coefficient for $\theta_{H V P}$ is positive (0.033) and the difference is statistically significant at $0.1 \%$-level.

3. The coefficient for per is positive (0.095) and the difference is statistically significant at $1 \%$-level.

\section{Result 6. (LVPs' behavior at the participation stage)}

1. Saying "no auction" at the participation stage for LVPs under ACAPS is more frequently taken than under SPAPS.

2. When LVPs' valuations increase, saying "auction" at the participation stage is more frequently taken.

3. As the period progresses, saying "no auction" at the participation stage for LVPs is more frequently taken.

Support. 1. In Table 5, the coefficient for $d_{A C A P S}$ is negative $(-1.098)$ and the difference is statistically significant at $0.1 \%$-level, which indicates the reduction that LVPs say "auction" under ACAPS.

2. The coefficient for $\theta_{L V P}$ is positive (0.024) and the difference is statistically significant at $0.1 \%$-level.

3. The coefficient for per is negative $(-0.098)$ and the difference is statistically significant at $0.1 \%$-level. 
Table 6: Players' behavior at the auction stage.

\begin{tabular}{lcc}
\hline & SPAPS & ACAPS \\
\hline Auction stage & $22.5 \%=180 / 800$ & $12.8 \%=102 / 800$ \\
$(1)$ HVPs win the auction & $63.3 \%=114 / 180$ & $82.4 \%=84 / 102$ \\
$(1 a)$ and $b_{S H B} \in\left[0, \theta_{L V P}-2\right)^{a}$ & $28.1 \%=32 / 114$ & $21.4 \%=18 / 84$ \\
$(1 b)$ and $b_{S H B} \in\left[\theta_{L V P}-2, \theta_{L V P}+2\right]$ & $19.3 \%=22 / 114$ & $45.2 \%=38 / 84$ \\
$(1 c)$ and $b_{S H B} \in\left(\theta_{L V P}+2,270\right]$ & $52.6 \%=60 / 114$ & $33.3 \%=28 / 84$ \\
$(2)$ LVPs win the auction & $36.7 \%=66 / 180$ & $17.6 \%=18 / 102$ \\
$(2 a)$ and $b_{S H B} \in\left[0, \theta_{H V P}-2\right)$ & $69.7 \%=46 / 66$ & $88.9 \%=16 / 18$ \\
$(2 b)$ and $b_{S H B} \in\left[\theta_{H V P}-2, \theta_{H V P}+2\right]$ & $18.2 \%=12 / 66$ & $0.0 \%=0 / 18$ \\
$(2 c)$ and $b_{S H B} \in\left(\theta_{H V P}+2,270\right]$ & $12.1 \%=8 / 66$ & $11.1 \%=2 / 18$ \\
\end{tabular}

${ }^{a} b_{S H B}$ : the second highest bid (or the first drop-out price)

\subsubsection{The Auction Stage}

Table 6 provides the distributions of players' bids under SPAPS and ACAPS. In order to analyze whether HVPs and LVPs bid rationally, we divide the regions of the secondhighest bid under SPAPS (or the first drop-out price under ACAPS), $b_{S H B}$, into three cases: (1) $b_{S H B}$ is less than loser's valuation minus 2 francs, (2) $b_{S H B}$ is in the interval between loser's valuation plus 2 francs and minus 2 francs, and (3) $b_{S H B}$ is more than loser's valuation plus 2 francs. Notice that we would like to analyze whether loser's bid is her own valuation (or quit when the price is her own valuation) of the equilibrium price within 2 francs as in Elbittar and Di Giannatale (2017) and Li (2017).

First, we investigate the behavior of LVPs. HVPs win the auction at $63.3 \%=114 / 180$ under SPAPS and at $82.4 \%=84 / 102$ under ACAPS. When we focus on the cases where the auction stage is proceeded and HVPs win the auction, $b_{S H B}$ is less than loser's valuation minus 2 francs at $28.1 \%=32 / 114$ under SPAPS and at 21.4\%=18/84 under ACAPS; $b_{S H B}$ is between loser's valuation plus 2 francs and minus 2 francs at $19.3 \%=$ $22 / 114$ under SPAPS and at $45.2 \%=38 / 84$ under ACAPS; $b_{S H B}$ is more than loser's valuation plus 2 francs at $52.6 \%=60 / 114$ under SPAPS and at 33.3\% $=28 / 84$ under ACAPS. Note that the number of the players who proceed to the auction under ACAPS 
$(102 / 800)$ is much less that under SPAPS (180/800). Even though, not only the aggregate proportion of the LVPs who bid between loser's valuation plus 2 francs and minus 2 francs under ACAPS $(45.2 \%=38 / 84)$ but also the number of the LVPs itself are greater than under SPAPS $(19.3 \%=22 / 114)$. In addition, not only the aggregate proportion of the LVPs who bid more than loser's valuation plus 2 francs under ACAPS $(33.3 \%=28 / 84)$ but also the number of the LVPs itself are less than under SPAPS $(52.6 \%=60 / 114)$.

Next, we investigate the behavior of HVPs. LVPs win the auction at $63.3 \%=114 / 180$ under SPAPS and at $82.4 \%=84 / 102$ under ACAPS. When we focus on the cases where the auction stage is proceeded and LVPs win the auction, they win the auction at $36.7 \%=$ $66 / 180$ under SPAPS and at $17.6 \%=18 / 102$ under ACAPS; $b_{S H B}$ is less than loser's valuation minus 2 francs at $69.7 \%=46 / 66$ under SPAPS and at 88.9\%=16/18 under ACAPS; $b_{S H B}$ is between loser's valuation plus 2 francs and minus 2 francs at $18.2 \%=$ $12 / 66$ under SPAPS and at $0.0 \%=0 / 18$ under ACAPS; $b_{S H B}$ is more than loser's valuation plus 2 francs at $12.1 \%=8 / 66$ under SPAPS and at $11.1 \%=2 / 18$ under ACAPS. In contrast to the results for LVPs, the aggregation proportions of the HVPs who bid less than loser's valuation minus 2 francs under SPAPS $(69.7 \%=46 / 66)$ and ACAPS $(88.9 \%=16 / 18)$ are large. Note that even though, most HVPs do not say "auction" at the participation stage under both mechanisms as in Section 4.3.1. This tendency is different from the results of experiments regarding the second-price auction and an ascending auction (e.g., Li, 2017). Then, this tendency seems to be come from the reason that two types of high values and low values are assigned and players know their types.

\section{Conclusion}

We first investigated a mechanism for King Solomon's Dilemma theoretically. While the mechanism of Mihara (2012) includes the second-price auction, we changed it into an ascending clock auction. By this modification, we had the result that a modified Mihara's mechanism implements the fist-best allocation in iterative elimination of obviously dominated strategies.

We experimentally compared the performance of Mihara's mechanism and a modified Mihara's mechanism for King Solomon's Dilemma. Our findings were as follows: (1) ACAPS attained the first-best allocation more frequently than SPAPS (Result 1); ${ }^{14}$ (2) ACAPS allocated the object to the right player more frequently than SPAPS (Result 2); (3) SPAPS was more inefficient than ACAPS from the view point of resource

\footnotetext{
${ }^{14}$ For both mechanisms, the first-best allocations and the right-player allocations were more frequently achieved in the latter half than in the first half.
} 
inefficiency and right-player inefficiency (Results 3 and 4); (4) Net mean efficiency under ACAPS was $91.3 \%$, while net mean efficiency under SPAPS was $71.8 \%$ (Table 2); and (5) The equilibrium strategies were frequently taken under ACAPS than under SPAPS (Section 4.3).

Although the performance of ACAPS was relatively better than that of SPAPS in our experiment, there are still open questions. The following are three instances; the participation fee, the number of players, and the types of players.

First, in our experiment, when LVPs' valuations increase, the proportions of the inefficiency of the monetary resources and the inefficiency regarding the wrong-player allocations increases, as in Subsection 4.2. A possible explanation for it is that if an LVP's valuation for the object is higher than the participation fee, he considers the case that he can get the object with payment which is lower than her valuation in the auction stage. Thus, we expect that increasing the participation fee may lower the rate of the choices of "auction" selected by LVPs at the participation stage. Conversely, we would like to investigate whether decreasing the participation fee may lower the rate of the choices of "no auction" selected by LVPs at the participation stage.

Second, in our experiment, there are only two players. If the number of players increases, then it may be harder for players to find the equilibrium strategies. Even in this case, we expect that the relative performance of our mechanism may be better than that of Mihara's mechanism.

Third, in our experiment, the two types of players were fixed, as in Elbittar and Di Giannatale (2017). The reason for this setting is that we would like to find whether there is learning effect or not. While we found learning effect as in Sections 4.2 and 4.3, a few subjects with low valuations (two subjects out of total 20 subjects with low valuations under each of SPAPS and ACAPS) participated the auctions to spite their counterparts with high valuations. ${ }^{15}$ This is because subjects' types were fixed so that subjects with low valuations envied their counterparts. ${ }^{16}$ On the other hand, in the experiment of Ponti, Gantner, López-Pintado, and Montgomery (2003), the two types of players were

\footnotetext{
${ }^{15}$ This observation is come from the post-experimental questionnaires. While the subjects who spited their counterparts understood that saying "no auction" is the best response for theirselves, they participated the auctions as their counterparts had to pay money including the participation fee.

${ }^{16}$ The subjects who spited their counterparts might not very much consider their own payoffs. As in our instructions, if subjects got negative points, the minimum hourly payment of 1013 Japanese yen was guaranteed. Even though, if they did not participate in the auction, they could get the larger amount of money. Then, our setting regarding how to calculate the final payment from subjects' payoffs gave the incentives to get the much larger amount of payoffs to the subjects. In short, while they had the chance to get larger amount of money, they might have decided to spite their counterparts since the loss of money by this behavior is not large.
} 
not fixed. If we follow this setting, each subject may change her strategy because he can be either an HVP or an LVP.

\section{References}

Barberà, S. (2011): "Strategyproof Social Choice," in Handbook of Social Choice and Welfare, vol. 2, pp. 731-831. Elsevier.

Costa-Gomes, M., V. P. Crawford, and B. Broseta (2001): "Cognition and Behavior in Normal-Form Games: An Experimental Study," Econometrica, 69(5), 1193-1235.

Elbittar, A., and S. Di Giannatale (2017): "Neither I nor You Shall Have Him": An Experimental Study of the King Solomon's Dilemma," Journal of Behavioral and Experimental Economics, 70, 55-69.

Fischbacher, U. (2007): "z-Tree: Zurich Toolbox for Ready-Made Economic Experiments," Experimental Economics, 10(2), 171-178.

Glazer, J., and C.-T. A. Ma (1989): "Efficient Allocation of a "Prize"-King Solomon's Dilemma," Games and Economic Behavior, 1(3), 222-233.

Kagel, J. H., R. M. Harstad, and D. Levin (1987): "Information Impact and Allocation Rules in Auctions with Affiliated Private Values: A Laboratory Study," Econometrica, 55(6), 1275-1304.

Katok, E., M. Sefton, and A. Yavas (2002): "Implementation by Iterative Dominance and Backward Induction: An Experimental Comparison," Journal of Economic Theory, 104(1), 89 - 103.

Kneeland, T. (2015): "Identifying Higher-Order Rationality," Econometrica, 83(5), 2065-2079.

Li, S. (2017): "Obviously Strategy-Proof Mechanisms," American Economic Review, 107(11), 3257-3287.

Masuda, T., Y. Okano, and T. Saijo (2014): "The Minimum Approval Mechanism Implements the Efficient Public Good Allocation Theoretically and Experimentally," Games and Economic Behavior, 83, 73-85.

Mihara, H. R. (2012): “The Second-Price Auction Solves King Solomon's Dilemma," Japanese Economic Review, 63(3), 420-429. 
Moore, J. (1992): "Implementation, Contracts, and Renegotiation in Environments with Complete Information," in Advances in Economic Theory: Sixth World Congress, ed. by J.-J. Laffont, vol. 1, pp. 182-281. Cambridge University Press, Cambridge, United Kingdom.

Nagel, R. (1995): "Unraveling in Guessing Games: An Experimental Study," American Economic Review, 85(5), 1313-1326.

Olszewski, W. (2003): "A Simple and General Solution to King Solomon's Problem," Games and Economic Behavior, 42(2), 315-318.

Perry, M., and P. J. Reny (1999): "A General Solution to King Solomon's Dilemma," Games and Economic Behavior, 26(2), 279-285.

Ponti, G., A. Gantner, D. López-Pintado, and R. Montgomery (2003): "Solomon's Dilemma: An Experimental Study on Dynamic Implementation," Review of Economic Design, 8(2), 217-239.

Qin, C.-Z., and C.-L. Yang (2009): "Make a Guess: A Robust Mechanism for King Solomon's Dilemma," Economic Theory, 39(2), 259-268.

Rapoport, A., and W. Amaldoss (2000): "Mixed Strategies and Iterative Elimination of Strongly Dominated Strategies: An Experimental Investigation of States of Knowledge," Journal of Economic Behavior \& Organization, 42(4), 483-521.

Sefton, M., and A. Yavas (1996): "Abreu-Matsushima Mechanisms: Experimental Evidence," Games and Economic Behavior, 16(2), 280-302. 